\title{
Quercetin sensitizes glioblastoma to t-AUCB by dual inhibition of Hsp27 and COX-2 in vitro and in vivo
}

\author{
Junyang Li ${ }^{1 * \dagger}$, Chao Tang ${ }^{1 \dagger}$, Liwen $\mathrm{Li}^{1}$, Rujun $\mathrm{Li}^{2}$ and Youwu Fan ${ }^{{ }^{*}}$
}

\begin{abstract}
Background: Evidences indicate that inflammatory process plays pivotal role in tumor disease. Soluble epoxide hydrolase inhibitors (sEHIs) have been shown to participate in anti-inflammation and tumorigenesis by protecting epoxyeicosatrienoic acids (EETs). Although we have previously revealed some effects of t-AUCB on glioma in vitro, further investigations are needed to demonstrate its effects on glioblastoma growth in vivo and how to strengthen its antitumor effect.

Methods: CCK-8 kit was used to test cell growth. Cell migration capacity was performed by wound healing assays. Transwell assay was used to test cell invasion potency. Cell-cycle analysis and cell apoptosis was performed by flow cytometry. The activity of caspase-3 in cells was measured using caspase-3 activity assay kits. Total RNA was extracted from cells lysated by TRIzol reagent. qRT-PCR was performed by ABI 7500 fast RT- PCR system. Lipofectamine RNAiMAX Transfection Reagent (Invitrogen) was used for siRNA transfection. Western blootting was used to test protein expression. Tumor cell xenograft mouse models were used for in vivo study. The SPSS version 17.0 software was applied for statistical analysis.
\end{abstract}

Results: Our data shown that t-AUCB inhibits cell proliferation, migration and invasion and induces cell cycle G1 phase arrest in vitro but induces no cell apoptosis; increased Hsp27 activation and following COX-2 overexpression confer resistance to t-AUCB treatment in glioblastoma both in vitro and in vivo; quercetin sensitizes glioblastoma to t-AUCB by dual inhibition of Hsp27 and COX-2 in vitro and in vivo.

Conclusions: These results indicate that combination of t-AUCB and quercetin may be a potential approach to treating glioblastoma.

Keywords: Glioma, Soluble epoxide hydrolase, Heat shock protein 27, Cyclooxygenase 2, Inhibitor

\section{Background}

Glioblastoma is the most common primary malignant tumor of the central nervous system in adults, which is highly aggressive and neurologically destructive. Despite the advances in surgery, radiotherapy and chemotherapy, survival time for patients with glioblastoma has remained at less than one year, not to mention the patients' pain and heavy economic burden [1-5]. In view of the impossibility of real total resection of glioblastoma in surgery, and

\footnotetext{
*Correspondence: ljy_njmu@126.com; fanyouwu_njzy@126.com

${ }^{\dagger}$ Equal contributors

${ }^{1}$ Department of Neurosurgery, Jinling Hospital, School of Medicine, Nanjing University, 305 East Zhongshan Road, Nanjing City 210002 Jiangsu Province China

Full list of author information is available at the end of the article
}

the serious side effects and the limited accessibility of radiotherapy, we suggest developing more efficient agent or combination of agents with great therapeutic effects and fewer side effects to treat glioblastoma or apply as postoperative adjuvant via circulatory system.

Recently, inflammation has been widely studied in malignant tumors and considered to participate in networks of activated signaling cascades, transcription factors and their coordinated interactions and promote tumorigenesis [6-8]. It could be effective therapy against malignant tumors to inhibit inflammation and then target inflammationmediated transcription-factor interplay and signaling pathways [9]. Epoxyeicosatrienoic acids (EETs), a metabolite converted from arachidonic acid (ARA) by cytochrome 
P450 (CYP) epoxygenases, have been reported as mediators with antihypertensive, anti-inflammatory, analgesic, and cardioprotective effects [10]. EETs are easily to be hydrolyzed in vivo by soluble epoxide hydrolase (sEH) to form it's less active or inactive metabolite dihydroxyeicosatrienoic acids (DHETs). Thus, various pharmacological inhibitors of sEH (sEHIs) have been developed to stabilize endogenous EETs and exert therapeutic effects [11]. Several studies have demonstrated that $\mathrm{sEH}$ play critical roles in angiogenesis and tumorigenesis, indicating the antitumor effects of sEHIs [12-14].

Our previous study has determined that $\mathrm{t}$-AUCB, an improved sEHi synthesized and kindly provided by Prof. Hammock and his team, inhibits human glioblastoma cell growth, although cells then acquire apoptosis-resistance to t-AUCB via Hsp27 activation [15]. Considering the well proved antihypertensive, anti-inflammatory and analgesic effects of sEHIs which may greatly alleviate the pain of the patients, we suggest sEHIs may be a potential agent for glioblastoma treatment and worth further study. Recently, Prof. Hammock and his team demonstrated that a combination of COX-2 inhibitor and sEH inhibitor (t-AUCB) synergistically inhibits primary tumor growth. They also developed a COX-2/sEH dual inhibitor, PTUPB, which significantly suppresses primary tumor growth and metastasis [16]. In present study, we study the effects and interactions of Hsp27 inhibitor, quercetin, and t-AUCB on glioblastoma cells, and demonstrate that combination of quercetin and $\mathrm{t}$-AUCB synergistically inhibits glioblastoma growth in vitro and in vivo. We also unexpectedly reveal that quercetin suppress COX-2 expression by Hsp27 inhibition and act as both COX-2 and Hsp27 inhibitor.

\section{Methods}

\section{Regents}

The sEH inhibitor t-AUCB was granted from Professor Bruce D. Hammock (Department of Entomology and UCD Cancer Research Center, University of California, Davis, CA, USA) [17], and the chemical constitution of t-AUCB was shown in Fig. 1a. The p38 MAPK inhibitor SB203580 and Hsp27 inhibitor quercetin were both purchased from Sigma-Aldrich (St. Louis, MO, USA). All of agents were dissolved in dimethyl sulfoxide (DMSO). The concentration which was never exceeded $0.1 \%(\mathrm{v} / \mathrm{v})$ was diluted in serum-supplemented medium immediately before use. For cell growth assays, cells were treated with $\mathrm{t}-\mathrm{AUCB}$ in different final concentrations of $0,10,50,100,150,200,300$ or $400 \mu \mathrm{M}$, and quercetin in final concentrations of $0,5,15$, 30 or $60 \mu \mathrm{M}$. For p38 MAPK inhibition, cells were treated with SB203580 in $20 \mu \mathrm{M}$ as we have described previously [15]. For Hsp27 inhibition, cells were treated with quercetin in $30 \mu \mathrm{M}$. In animal experiments, the mice were treated with $\mathrm{t}$-AUCB $\left(3 \mathrm{mg} \cdot \mathrm{kg}^{-1} \cdot \mathrm{d}^{-1}\right)$ by oral gavage or/and injected intraperitoneally with $20 \mathrm{mg} / \mathrm{kg}$ quercetin every day.

\section{Cell culture}

Human glioblastoma cell lines U251 and U87 were provided by ATCC (American Type Culture Collection) as we used previously [15]. All cells were cultured in Dulbecco's Modified Eagle's Medium (DMEM) supplemented with $10 \%$ fetal bovine serum (FBS) and $1 \%$ penicillin and streptomycin (complete medium). Cells were maintained at $37{ }^{\circ} \mathrm{C}$ in a humidified atmosphere of $95 \%$ air and $5 \%$ $\mathrm{CO}_{2}$.

\section{Cell growth assay}

Cell growth ability was exhibited by the cell counting kit-8 (CCK-8) from Dojindo Laboratories (Kumamoto, Japan). Cells were transplanted into a 96-well plate with density of 5000 cells/well and then treated differently. Cells were cultured in humidified incubator containing $5 \% \mathrm{CO} 2$ and $95 \%$ air. 48 hours later, culture medium in each well was discarded, and $100 \mu \mathrm{l}$ fresh serum-free medium contained $10 \mu \mathrm{l}$ CCK-8 solution was added into each well. After two hour incubation, the optical density value (absorbance) was recorded at $450 \mathrm{~nm}$ using an enzyme-linked immunosorbent assay plate reader (Bio-Rad Laboratories, Inc., Berkeley, CA, USA).

\section{Cell migration and invasion assays}

Cell migration capacity was performed by wound healing assays. U251 cells were transplanted in 6-well plates. The scratch wounds were generated with a pipette tip. Cells were washed with PBS and incubated in a serumfree medium. 16 hours after scratching, wounds were fixed and photographic images were collected. Transwell assay was used to test cell invasion potency. U87 cells were transplanted in 24-well BioCoat Matrigel Invasion Chambers ( $8 \mu \mathrm{m}$ pore size, Corning) with matrigel-coated in serum-free DMEM. Conditioned medium were cultured into the lower chambers like chemo-attractants. After incubation for 24 hours, the cells from upper surface of filters were scraped by a cotton swab, whereas matrigel were penetrated by other cells which adherent to the bottom of membrane. Methanol was used as fixed solution and $0.1 \%$ crystal violet were used to stain.

\section{Cell-cycle analysis by flow cytometry}

The cells were seeded in $10 \mathrm{~cm}$ culture dishes, then treated separately after adherence for $48 \mathrm{~h}$ at $37{ }^{\circ} \mathrm{C}$ in humidified incubator. Cells were collected followed by trypsinization, centrifuged (3500 rpm for $5 \mathrm{~min}$ ), and washed twice with PBS. $1 \mathrm{ml} 70 \%$ ethanol was used as fixed solution, precipitated by centrifugation $(3500 \mathrm{rpm}$ for $5 \mathrm{~min}$ ), and propidium iodide was used for staining 
A<smiles>O=C(NC12CC3CC(CC(C3)C1)C2)N[C@H]1CC[C@H](Oc2ccc(C(=O)O)cc2)CC1</smiles><smiles>O=C(NC1CCC(Oc2ccc(C(=O)O)cc2)CC1)NC12CC3CC(CC(C3)C1)C2</smiles>

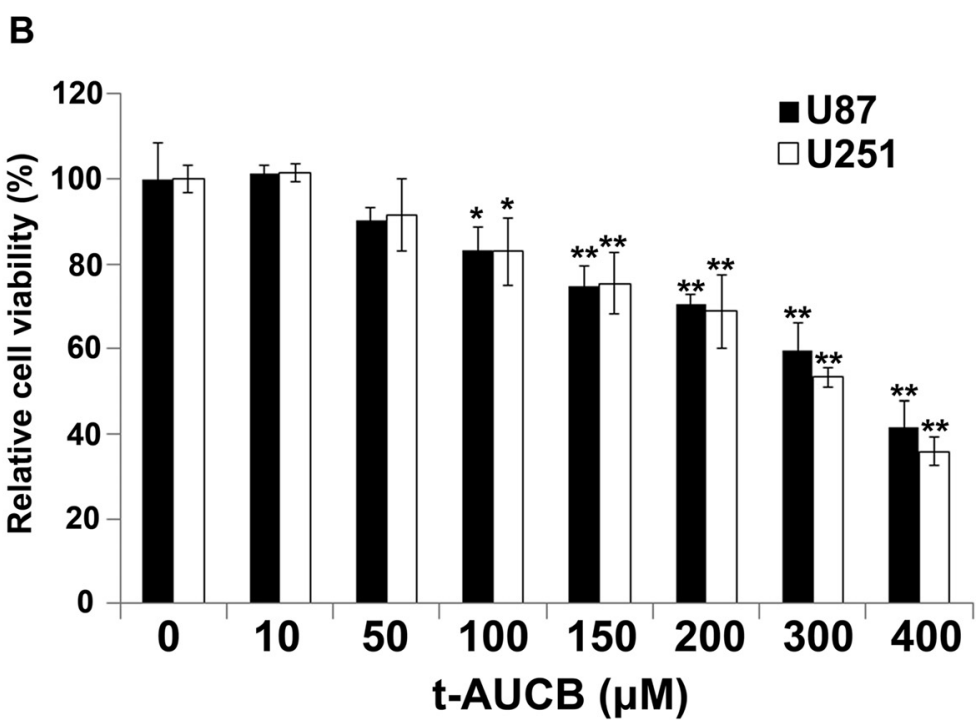

Fig. 1 t-AUCB inhibits cell proliferation. a: The chemical constitution of t-AUCB. $\mathbf{b}$ : The percentage of OD value in t-AUCB treated cells compare with control represent the relative cell viability (\%) in CCK-8 assay. U87 and U251 cells were treated with vehicle control (0 $\mu \mathrm{M}), 10 \mu \mathrm{M}, 50 \mu \mathrm{M}, 100 \mu \mathrm{M}, 150 \mu \mathrm{M}$, $200 \mu \mathrm{M}, 300 \mu \mathrm{M}$ or $400 \mu \mathrm{M}$ t-AUCB for $48 \mathrm{~h}$. t-AUCB suppresses cell proliferation in a concentration-dependent manner since $100 \mu \mathrm{M}\left({ }^{*} P<0.05\right) .150 \mu \mathrm{M}$ to $400 \mu \mathrm{M}$ t-AUCB causes more efficient cell growth inhibition $(* * 0.01)$

nuclei. A total of 10,000 nuclei were analyzed in a FACSCalibur flow cytometer (BD Biosciences, San Jose, CA, USA).

\section{Apoptosis analysis by flow cytometry}

For apoptosis analysis, the cells were seeded in $10 \mathrm{~cm}$ culture dishes, then treated separately after adherence for $48 \mathrm{~h}$ at $37{ }^{\circ} \mathrm{C}$ in humidified incubator. Cells were collected followed by trypsinization, centrifuged $(3500 \mathrm{rpm}$ for $5 \mathrm{~min}$ ), and washed twice with PBS, the supernatant was discarded. The pellet was incubated away from light for $15 \mathrm{~min}$ at room temperature with Annexin V-fluorescein isothiocyanate (FITC) and propidium iodide (PI) before analysis with a FACSAria III flow cytometer (BD Biosciences, San Jose, CA, USA) according to the standard protocol.

\section{Caspase-3 activity assay}

As we previously described [15], the activity of caspase-3 in cells was measured using caspase- 3 activity assay kits from Millipore (Kankakee, IL, USA) following the manufacturer's instructions. Absorbance was represented the level of caspase- 3 activity and tested at $405 \mathrm{~nm}$ in a microtiter plate reader. In a word, cell samples were collected and resuspend in chilled $1 \times$ Cell Lysis Buffer for $10 \mathrm{~min}$, then centrifuged at $4{ }^{\circ} \mathrm{C}$ for $5 \mathrm{~min}$ in a microcentrifuge $(10,000 \times g)$. The supernatants were subsequently added into a 96-well plate and incubated with Ac-DEVD-pNA in the working solution containing caspase- 3 substrate for $1 \mathrm{~h}$ at $37^{\circ} \mathrm{C}$. Fold-increase in caspase- 3 activity was determined by comparing the absorbance from an apoptotic sample with an un-induced control after subtracting the background value reading from cell lysates and buffers. Each determination was performed in triplicate. 
RNA extraction and Quantitative real-time PCR

Total RNA was extracted from cells lysated by TRIzol reagent (Invitrogen, USA) according to the manufacturer's instructions. The quality and quantity of the RNA purity were assessed by spectrophotometer and standard electrophoresis. cDNA was synthesized from $1 \mu \mathrm{g}$ RNA and reverse transcribed using PrimerScript ${ }^{\mathrm{TM}}$ RT reagent Kit (Takara). The expression levels were quantified by Taq$\operatorname{man}^{\mathrm{Tm}}$ Assay kit (Applied Biosystems, USA) in according with the manufacturer's instructions. The sequences of primers for the COX-2 were as follows: forward: 5'ATACCAAAACCGCATTGCCG-3'; reverse: 5'- TCTAAC TCCGCAGCCATTTC-3'. Expression of GAPDH was performed as normalized. The sequences of primers for GAPDH were as follows: forward: 5'-GGAAGGTGAAGG TCGGAGTC-3'; reverse: 5'-GTCTTCTGGGTGGCAGTGAT-3'. Each reaction was performed by ABI 7500 fast RTPCR system in triplicate. The expression of gene was defined based on the threshold cycle $(\mathrm{Ct})$ value. The relative expression of the studied samples were assessed using the comparative delta-delta $\mathrm{Ct}$ method (TaqMan Relative Quantification Assay software), adjusted to GAPDH expression level.

\section{RNA interference}

Cells were grown in 10-cm culture dish and transiently transfected with Hsp27 or COX-2 specific small interfering RNA (siRNA). The siRNA oligos of Hsp27 (sc-29350), COX-2 (sc-270376) and the negative control siRNAs were purchased from Santa Cruz Biotechnology (Dallas, TX, USA). Lipofectamine RNAiMAX Transfection Reagent (Invitrogen) was used for siRNA transfection according to the manufacturer's protocol.

\section{Western blot analysis}

Cell protein was lysates in ice-cold RIPA buffer (Beyotime Institute of Biotechnology, Shanghai, China) containing with Phenylmethanesulfonyl fluoride (PMSF) and protease inhibitor cocktail. The procedure of Western Blot was described as before [15]. The whole cell lysates were separated by SDS-polyacrylamide gel electrophoresis (SDSPAGE) and transferred to a polyvinylidene fluoride membrane (Millipore Corporation, Bedford, MA, USA). All membranes were probed with primary antibodies after $4{ }^{\circ} \mathrm{C}$ overnight, and followed by incubation with secondary antibody. Proteins were visualized with chemiluminescence luminol reagents (Beyotime Institute of Biotechnology, Shanghai, China). Antibodies against $\beta$-actin (\#3700), cMyc (\#9402), Cdc25A (\#3652), Cyclin D1 (\#2978), CDK4 (\#12790), CDK6 (\#3136), MCM3 (\#4012), MCM7 (\#3735), COX-2 (\#12282), Hsp27 (\#2402), p-Hsp27 (Ser78) (\#2405) were purchased from Cell Signaling Technology (Beverly, MA, USA). Public software ImageJ (National Institutes of
Health, USA) was used to quantify the densitometry of the immunoblotting bands.

\section{Tumor cell xenograft mouse models}

Animal experiments protocols were approved by the Institutional Animal Committee of Jinling Hospital. The BALB/c nude mice (male at 5 to 6 weeks old) were obtain from Department of comparative medicine (Jinling Hospital, China) and maintained in specific pathogen-free (SPF) conditions. Approximately $1.0 \times 10^{6}$ U87 cells were transplanted subcutaneously to develop a mouse xenograft model of human glioblastoma. Once tumor diameter reach to $2-4 \mathrm{~mm}$, the treatment with quercetin or/and t-AUCB was administered. The length and width of the tumors were measured every other day using vernier caliper, and tumor volume was calculated (tumor volume $=1 / 2 \times$ length $\times$ width $\left.^{2}\right)$. The mice were treated with $t$-AUCB (3 $\mathrm{mg} \cdot \mathrm{kg}^{-1} \cdot \mathrm{d}^{-1}$ ) by oral gavage or/and injected intraperitoneally with $20 \mathrm{mg} / \mathrm{kg}$ quercetin every day. 14 days after treatment, all tumors retrieved from animals were measured, weighed and submitted for western blot analysis for the expression of Hsp27, p-Hsp27 and COX-2.

\section{Statistical analysis}

All experiments were replicated in triplicate at least. The SPSS version 17.0 software (SPSS Inc., Chicago, IL, USA) was applied for statistical analysis. Comparisons between treated groups and vehicle control were performed using independent $t$ test, and expressed as mean \pm standard deviation (SD). $P<0.05$ were considered statistically significant.

\section{Results}

t-AUCB inhibits cell proliferation, migration and invasion The CCK- 8 assay kit was used to test the cell proliferation. Human glioblastoma cell lines U251 and U87 were treated with vehicle control (DMSO), $10 \mu \mathrm{M}, 50 \mu \mathrm{M}, 100 \mu \mathrm{M}$, $150 \mu \mathrm{M}, 200 \mu \mathrm{M}, 300 \mu \mathrm{M}$ or $400 \mu \mathrm{M}$ t-AUCB for $48 \mathrm{~h}$. As the results shown in Fig. 1, t-AUCB suppresses cell proliferation in a concentration-dependent manner since $100 \mu \mathrm{M}$ $(P<0.05)$. $150 \mu \mathrm{M}$ to $400 \mu \mathrm{M}$ t-AUCB causes more efficient cell growth inhibition $(P<0.01)$. The IC50 of t-AUCB is $347.38 \mu \mathrm{M}$ (to U87 cells) and $305.05 \mu \mathrm{M}$ (to U251 cells).

Cell migration was evaluated by wound healing analysis. U251 cells were planted and treated with vehicle control (DMSO), $10 \mu \mathrm{M}, 100 \mu \mathrm{M}$ or $200 \mu \mathrm{M}$ t-AUCB for 16 h. $100 \mu \mathrm{M}$ or $200 \mu \mathrm{M}$ t-AUCB treatment suppresses the scratched cell monolayer healing, whereas $10 \mu \mathrm{M}$ t-AUCB has no effect (Fig. 2a and b).

Cell invasion was analyzed using matrigel-coated transwell chambers. U87 cells were planted into the chambers and treated with vehicle control (DMSO), $10 \mu \mathrm{M}, 100 \mu \mathrm{M}$ or $200 \mu \mathrm{M}$ t-AUCB for $24 \mathrm{~h}$. Cells treated with $100 \mu \mathrm{M}$ or 




$200 \mu \mathrm{M}$ t-AUCB showed a low level of penetration through the membranes compared with those treated with DMSO or $10 \mu \mathrm{M}$ t-AUCB (Fig. 2c and d).

\section{t-AUCB induces cell cycle $\mathrm{G} 1$ phase arrest}

For cell cycle assay, U251 and U87 cells were treated with DMSO (vehicle control), $10 \mu \mathrm{M}$ or $150 \mu \mathrm{M}$ t-AUCB for $48 \mathrm{~h}$ followed by flow cytometric analysis. $150 \mu \mathrm{M} \mathrm{t}$-AUCB treated cells exhibited $\mathrm{G} 1$ phase proportion increase with $\mathrm{S}$ phase proportion decrease (Fig. 3a and b). Interestingly, $10 \mu \mathrm{M}$ t-AUCB induces increase of G1 phase percentage, although it does not induce cell growth inhibition.

To further investigate how $\mathrm{t}$-AUCB regulates cell cycle, we detected the regulators involved in G1/S phase cell cycle by western blot. In $150 \mu \mathrm{M}$ t-AUCB treated cells, the expression levels of c-Myc, Cdc25A, Cyclin D1, CDK4 and CDK6, which play key roles in G1-S entry, were decreased (Fig. 3c). Moreover, DNA replication factors, MCM3 and MCM7, were found to decrease in t-AUCB treated cells (Fig. 3d).
t-AUCB increases COX-2 expression by Hsp27 activation Our previous study has demonstrated that acquired Hsp27 activation confers resistance to t-AUCB in glioma cells [15]. Since COX-2 overexpression in gliomas is associated with poor prognosis [18], we hypothesized that COX-2 may participate in Hsp27 conferred resistance to t-AUCB. To test this hypothesis, we detected COX-2 mRNA level by qRT-PCR and its protein expression by western blot. Our data demonstrated that, t-AUCB treatment increases COX2 expression in both mRNA and protein level $(P<0.01)$ (Fig. 4a and b). With the blockage of Hsp27 activation by p38 MAPK inhibitor SB203580, t-AUCB-induced COX-2 overexpression was partially attenuated (Fig. 4c), indicating the role of Hsp27 activation in COX-2 increase. To further investigate the effect of Hsp27 on COX-2 expression, cells were pretreated with $\mathrm{Hsp} 27$ inhibitor quercetin or Hsp27 siRNA followed by t-AUCB treatment. In cells without $\mathrm{t}$-AUCB treatment, both Hsp27 protein expression and its phosphorylation level were suppressed by quercetin or Hsp27 siRNA, resulting in significant decrease of COX-2 expression (Fig. 4d). Interestingly, in 


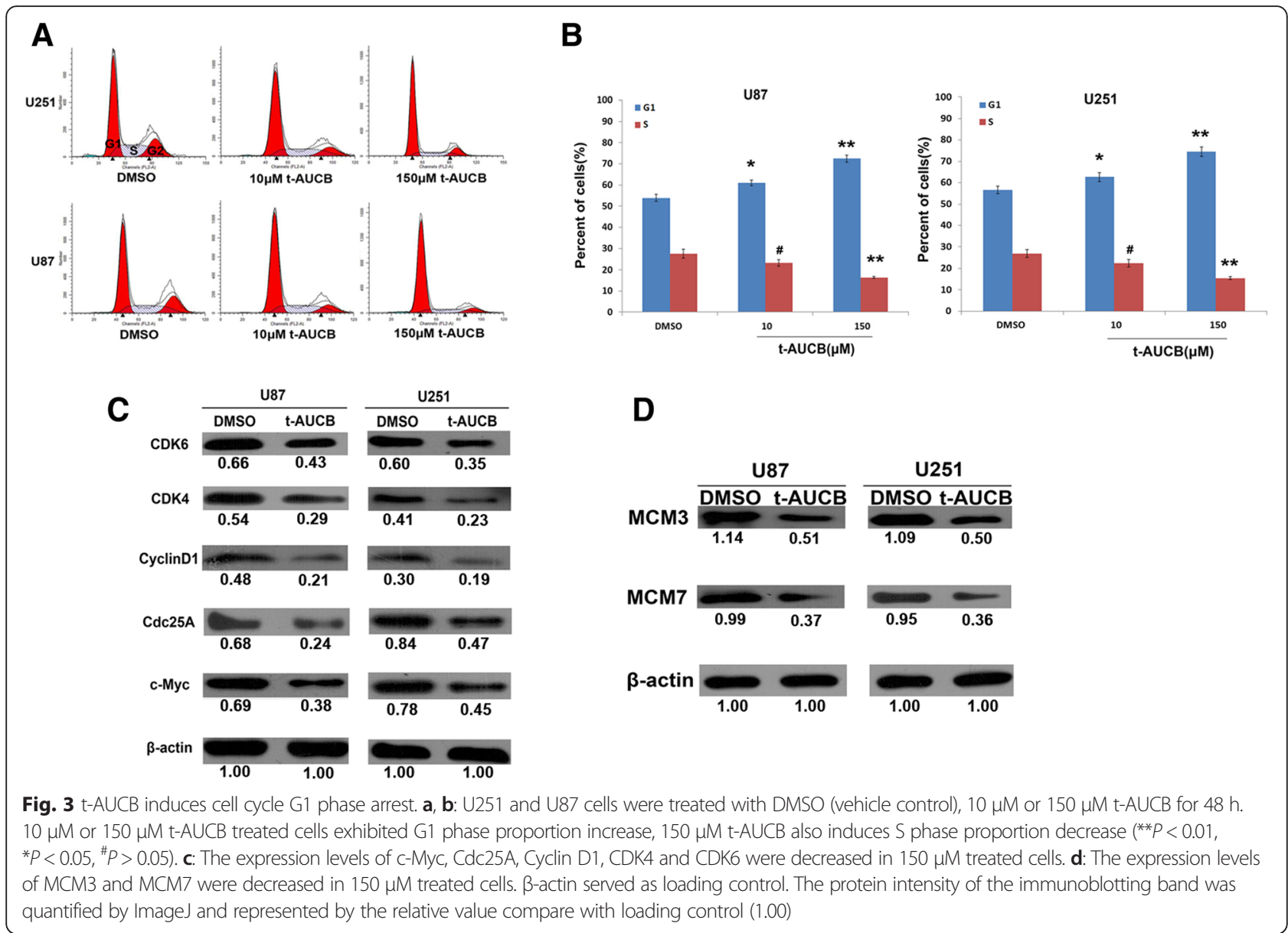

cells treated with quercetin plus t-AUCB, decrease of COX-2 and Hsp27 expression and Hsp27 phosphorylation was partially reversed (Fig. 4d).

\section{Quercetin strengthens t-AUCB-induced cell growth inhibition} by inhibition of Hsp27 and COX-2

Since either inhibition of Hsp27 or COX-2 has been reported to facilitate glioma cell death [18-20], we then investigate whether quercetin, which inhibits both Hsp27 and COX-2 expression, could strengthens t-AUCB-induced cell growth inhibition. U87 and U251 cells were separately treated with vehicle control, $5 \mu \mathrm{M}, 15 \mu \mathrm{M}, 30 \mu \mathrm{M}, 60 \mu \mathrm{M}$ quercetin, $200 \mu \mathrm{M}$ t-AUCB, or $200 \mu \mathrm{M}$ t-AUCB plus $15 \mu \mathrm{M}, 30 \mu \mathrm{M}$ or $60 \mu \mathrm{M}$ quercetin for $48 \mathrm{~h}$. Cell growth was tested using CCK-8 kit. As the results shown in Fig. 5a, quercetin inhibits cell growth in concentrationdepended manner since $15 \mu \mathrm{M}$, and strengthens $\mathrm{t}$-AUCB induced cell growth inhibition. Moreover, the treatment of $\mathrm{t}$-AUCB plus quercetin behaved more efficiently than quercetin alone $(P<0.01)$, indicating that $\mathrm{t}$-AUCB can also strengthen quercetin-induced cell death. To further determine the role of Hsp27 or COX-2 in cell growth inhibition, synthetic siRNA was transfected into cells to knockdown expression of Hsp27 or COX-2. Given the downregulation of Hsp27 or COX-2, t-AUCB induces more intense cell death $(P<0.01)$ (Fig. 5b). Interestingly, it seemed that cells with knockdown of Hsp27 are more sensitive to t-AUCB than those with knockdown of COX-2 $(P<0.05)$. These results demonstrated that quercetin sensitizes cells to t-AUCB by downregulation of $\mathrm{Hsp} 27$ and COX-2.

Our previous study has reported that $\mathrm{t}-\mathrm{AUCB}$ induces no significant cell apoptosis in U87 and U251 cells because of Hsp27 conferred resistance [15]. Herein, we investigated whether quercetin could reverse the apoptosis resistance by Hsp27 inhibition. U87 cells were treated with vehicle control, $200 \mu \mathrm{M}$ t-AUCB, $30 \mu \mathrm{M}$ quercetin or $200 \mu \mathrm{M}$ t-AUCB plus $30 \mu \mathrm{M}$ quercetin for $48 \mathrm{~h}$. Cell apoptosis was analyzed using flow cytometer. As the results shown in Fig. $5 \mathrm{c}$ and d, in DMSO (vehicle control) treated cells the apoptosis proportion (Low Right and Upper Right section) was $4.51 \pm 0.69 \%$. Cells treated with $200 \mu \mathrm{M}$ t-AUCB exhibited no increase in apoptosis proportion of $5.62 \pm 1.48 \%(P>0.05)$. Cells treated with $30 \mu \mathrm{M}$ quercetin also exhibited no increase in apoptosis proportion of $6.35 \pm 1.55 \%(P>0.05)$. For 


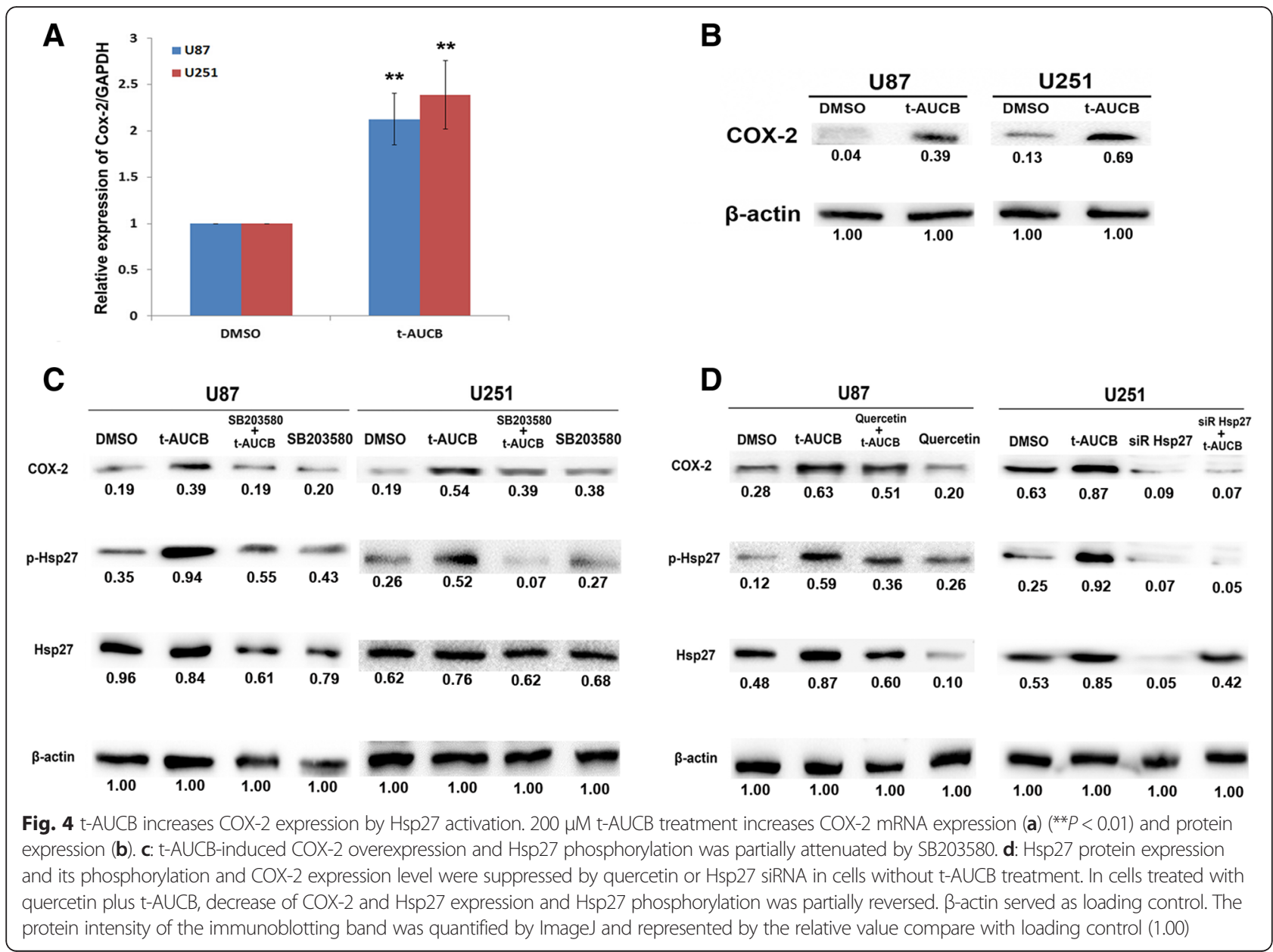

cells treated with $30 \mu \mathrm{M}$ quercetin plus $200 \mu \mathrm{M}$ t-AUCB, the apoptosis proportion was significantly increased into $16.38 \pm 1.53 \%(P<0.01)$. The caspase-3 activity was also tested in different treated cells, as the data shown, $\mathrm{t}-\mathrm{AUCB}$ plus quercetin significantly increases the activity of caspase$3(P<0.01)$ (Fig. 5e).

\section{Quercetin sensitizes glioblastoma to t-AUCB treatment in vivo}

To investigate the therapeutic effects of quercetin and t-AUCB on glioblastoma growth in vivo, we developed a mouse xenograft model of human glioblastoma by subcutaneously transplanting U87 cells in BALB/c nude mice. Once tumors grow to $2-4 \mathrm{~mm}$ in diameter, the treatment with quercetin or/and t-AUCB was administered as described in Materials and Methods section. The volume of xenograft tumor was measured and compared between different groups every two days up to 14 days. As the results shown in Fig. 6a and b, t-AUCB has no effect on tumor growth $(P>0.05)$. $\mathrm{t}-\mathrm{AUCB}$ plus quercetin treatment exhibits significant tumor inhibition, compared with control group $(P<0.01)$, t-AUCB alone group $(P<0.01)$ or quercetin treatment group $(P<0.05)$. Moreover, the weight of each tumor block was measured. The data confirmed the results from tumor volume measurement (Fig. 6c). We then determined the expression of Hsp27, p-Hsp27 and COX-2 in xenograft tumors with different treatment by western blot. The levels of Hsp27 phosphorylation and COX-2 protein were elevated in $\mathrm{t}$-AUCB treated tumors and sharply decreased in quercetin treated or quercetin plus t-AUCB treated tumors (Fig. 6d). These results suggested that $\mathrm{t}$-AUCB induces no tumor growth inhibition in vivo, although it inhibits cell growth in vitro. Quercetin, a Hsp27 inhibitor, sensitizes glioblastoma to t-AUCB treatment and causes significant tumor inhibition in vivo.

\section{Discussion}

We have demonstrated previously that acquired Hsp27 activation by p38 MAPK/MAPKAPK2/Hsp27 signaling cascade confers apoptosis-resistance to t-AUCB treatment in glioblastoma cells, although the cell proliferation inhibition and cell cycle $\mathrm{G} 1$ phase arrest induced by $\mathrm{t}$-AUCB were significant [15]. Actually, in the present study, we further demonstrated that t-AUCB suppresses not only cell proliferation but also cell migration and invasion, as well as the expression of cell cycle G1/S transition factors 

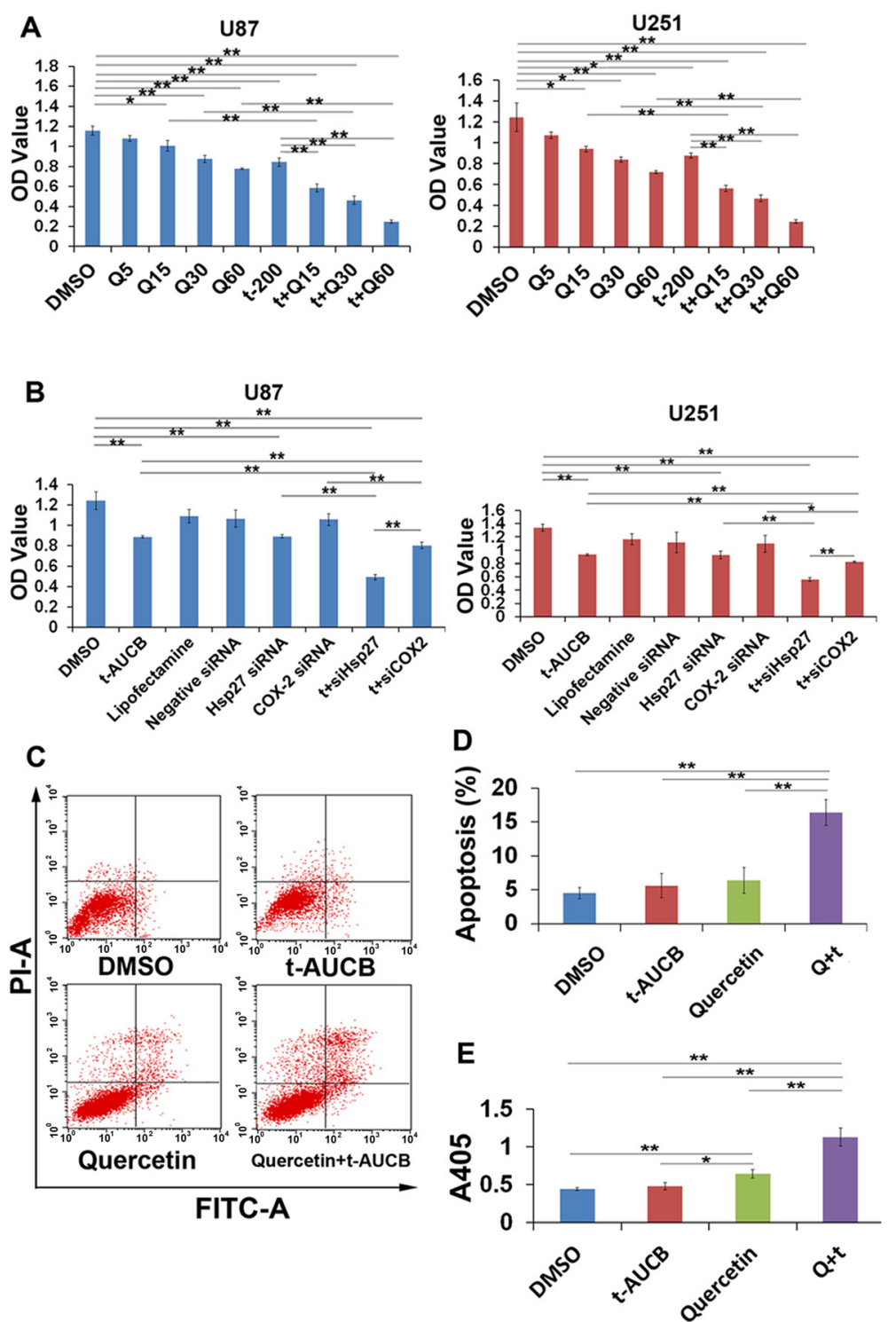

Fig. 5 Quercetin strengthens t-AUCB-induced cell growth inhibition by inhibition of Hsp27 and COX-2. a: CCK-8 assay for cell viability. U87 and U251 cells were separately treated with vehicle control (DMSO), $5 \mu \mathrm{M}, 15 \mu \mathrm{M}, 30 \mu \mathrm{M}, 60 \mu \mathrm{M}$ quercetin, $200 \mu \mathrm{M}$ t-AUCB, or $200 \mu \mathrm{M}$ t-AUCB plus $15 \mu \mathrm{M}, 30 \mu \mathrm{M}$ or $60 \mu \mathrm{M}$ quercetin for $48 \mathrm{~h}$. The OD values represented the cell viability. Quercetin inhibits cell growth in concentration-depended manner since $15 \mu \mathrm{M}$, and strengthens t-AUCB induced cell growth inhibition. t-AUCB also strengthens quercetin-induced cell death ${ }^{*} P<0.05$, $\left.{ }^{* *} P<0.01\right)$. Q: Quercetin; t: t-AUCB. $\mathbf{b}$ : CCK-8 assay for cell viability. In U87 and U251 cells, knockdown of Hsp27 or COX-2 strengthens t-AUCB-induced cell growth inhibition ( ${ }^{*} P<0.05,{ }^{*} P<0.01$ ). siHsp27: Hsp27 siRNA; siCOX-2: COX-2 siRNA; t: t-AUCB. c, d: Cell apoptosis analysis for U87 cells treated with vehicle control, $200 \mu \mathrm{M}$ t-AUCB, $30 \mu \mathrm{M}$ quercetin or $200 \mu \mathrm{M}$ t-AUCB plus $30 \mu \mathrm{M}$ quercetin for $48 \mathrm{~h}$. Vehicle control treated cells the apoptosis proportion (Low Right and Upper Right section) was $4.51 \pm 0.69 \%$. Cells treated with $200 \mu \mathrm{M}$ t-AUCB exhibited no increase in apoptosis proportion of $5.62 \pm 1.48 \%$ ( $P>0.05)$. Cells treated with $30 \mu \mathrm{M}$ quercetin also exhibited no increase in apoptosis proportion of $6.35 \pm 1.55 \%(P>0.05)$. For cells treated with $30 \mu \mathrm{M}$ quercetin plus $200 \mu \mathrm{M}$ t-AUCB, the apoptosis proportion was significantly increased into $16.38 \pm 1.53 \%$ (**P<0.01). Q: Quercetin; t: t-AUCB. e: The caspase-3 activity assay for U87 cells treated with vehicle control, $200 \mu \mathrm{M}$ t-AUCB, $30 \mu \mathrm{M}$ quercetin or $200 \mu \mathrm{M}$ t-AUCB plus $30 \mu \mathrm{M}$ quercetin. OD value represented the caspase-3 activity $\left.{ }^{*} P<0.05,{ }^{* *} P<0.01\right)$. Q: Quercetin; t: t-AUCB

and DNA replication factors. It seems that the effects of $\mathrm{t}$-AUCB on glioblastoma cells are complicated with the concomitance of cytotoxicity and anti-cytotoxicity. Zhang et al. recently demonstrated that high-dose t-AUCB $\left(10 \mathrm{mg} \cdot \mathrm{kg}^{-1} \cdot \mathrm{d}^{-1}\right)$ increases primary tumor growth and metastasis by stimulating tumor angiogenesis and VEGF levels, whereas low dose t-AUCB, $1 \mathrm{mg} \cdot \mathrm{kg}^{-1} \cdot \mathrm{d}^{-1}$ or $3 \mathrm{mg}$. $\mathrm{kg}^{-1} \cdot \mathrm{d}^{-1}$, inhibits Lewis lung carcinoma metastasis or has no effect on primary tumor growth and metastasis, which makes the effects of $\mathrm{t}$-AUCB on tumors more and more complicated [16]. SEHIs are considered to stimulate primary tumor growth and metastasis via EETs protection 


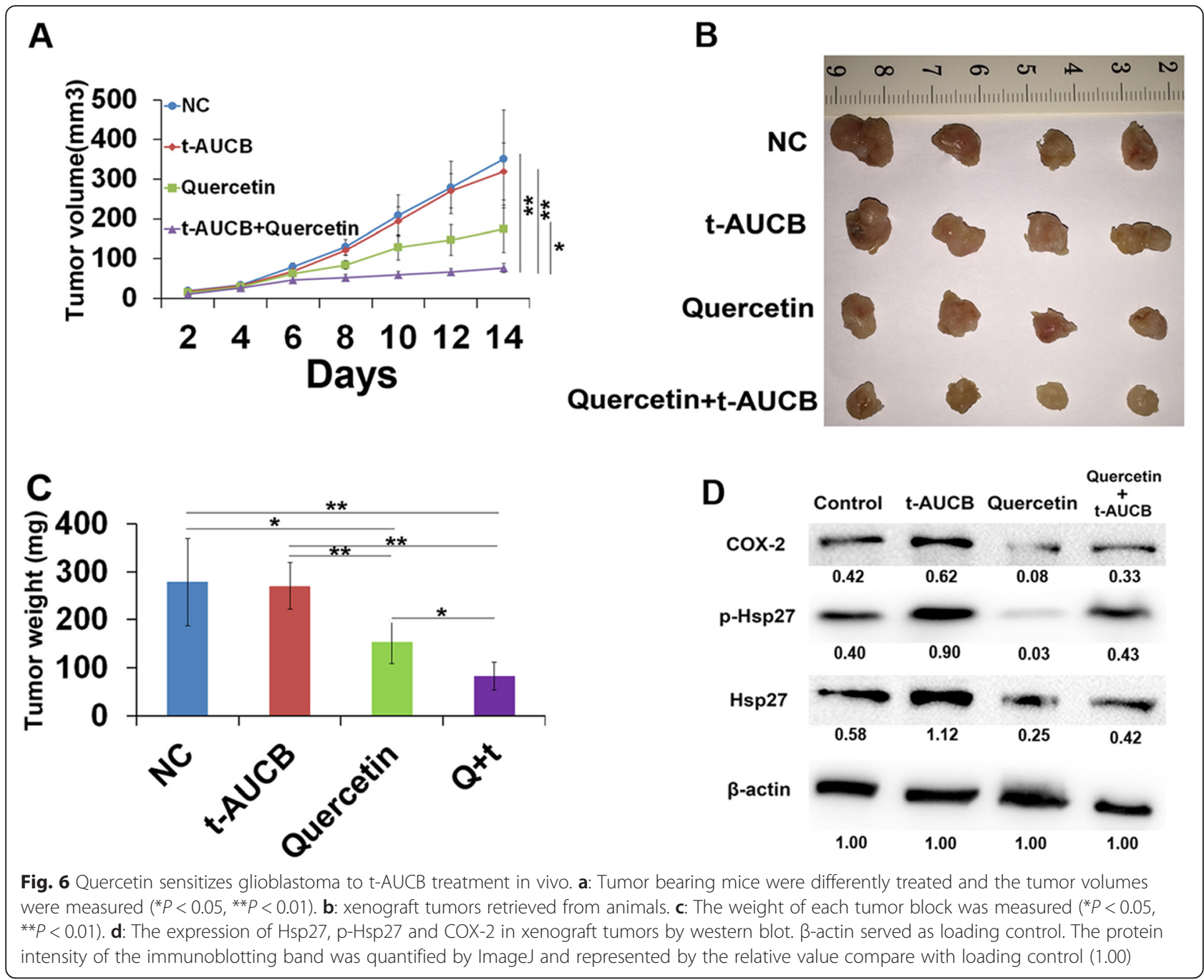

and following VEGF-dependent proangiogenic and protumorigenic effects $[21,22]$. However, COX-2 inhibition has been shown to inhibit VEGF production, indicating that EETs-induced angiogenesis and tumorigenesis may be reduced or eliminated by inhibition of COX-2 [23]. Moreover, sEHIs may further sensitize the anti-inflammatory and antiangiogenic effects of COX-2 inhibition [13, 16]. Zhang et al. then demonstrated that the combination of $\mathrm{t}-\mathrm{AUCB}$ and COX-2 inhibitor synergistically inhibits primary tumor growth and metastasis, although t-AUCB alone has no tumor inhibitory effect [16]. Previously, we have demonstrated similar results that combination of $\mathrm{t}-\mathrm{AUCB}$ and inhibitor of Hsp27 phosphorylation effectively induced cell apoptosis and synergistically inhibits cell growth in vitro. Thus, we hypothesized that COX-2 and Hsp27 may interactively participate in conferring resistance to t-AUCB.

Our present data revealed that t-AUCB increases COX-2 expression by Hsp27 activation which is partially reversed by SB203580, a p38 MAPK inhibitor. Then, quercetin or Hsp27 specific siRNA was pretreated to inhibit Hsp27 expression before t-AUCB treatment. Decreased Hsp27 expression and activation resulted in significant decrease of COX-2 expression in cells without t-AUCB treatment, whereas Hsp27 expression and activation and COX-2 expression were partially suppressed by quercetin in t-AUCB treated cells. These results suggested that quercetin suppresses Hsp27 and COX-2 expression but t-AUCB partially reverse quercetin-induced Hsp27 and COX-2 suppression. However, combination of quercetin and t-AUCB induces significant cell apoptosis and more efficient cell growth inhibition than each alone (Fig. 5), although cells treated with quercetin alone show the least expression of Hsp27 and COX-2. It seems that the increased Hsp27 activation and COX-2 expression contribute to acquire resistance to $\mathrm{t}$-AUCB treatment, and quercetin partially reverses the resistance by Hsp27 and COX-2 inhibition. The combination of quercetin and t-AUCB was also demonstrated to significantly inhibit glioblastoma growth in vivo from our data (Fig. 6). Although t-AUCB alone has no effect on xenograft glioblastoma, quercetin reverses the resistance of tumors 
to t-AUCB treatment and causes more efficient tumor growth inhibition than itself alone. Previously, combination of $\mathrm{t}-\mathrm{AUCB}$ and Hsp27 phosphorylation inhibitor has been reported to synergistically inhibit glioblastoma cell growth in vitro by us [15], and combination of t-AUCB and COX-2 inhibitor has been shown to synergistically inhibit primary tumor growth by others [16]. Here we demonstrated for the first time that combination of t-AUCB and quercetin with co-inhibition of Hsp27 and COX-2 synergistically inhibits glioblastoma growth in vitro and in vivo.

Few literatures have mentioned the interaction of Hsp27 and COX-2 in tumors. Lasa, et al. demonstrated that p38 MAPK/MAPKAPK2/Hsp27 signaling cascade regulates COX-2 mRNA stability in HeLa cells [24]. They found that COX-2 mRNA is stabilized by the activation of p38 MAPK, which is blocked by SB203580, and that the effects of p38 MAPK are mediated by phosphorylation of Hsp27 [24]. Our present study demonstrated similar effect that COX-2 mRNA and protein expression is increased by $\mathrm{Hsp} 27$ activation. However, it is impossible to apply SB203580 or Hsp27 siRNA in clinical medication; thus, we decided to test the effects of Hsp27 inhibitor quercetin which had been studied in clinical trials on in vitro and in vivo system [25]. Quercetin, a bioflavonoid widely distributed in plants, is well known as one of the "nature agents" with antitumor effects [26, 27]. It has been demonstrated to inhibit Hsp27 and display antitumor activity in several tumor cells or cancer stem cells, such as lung cancer stem cells [28], breast cancer stem cells [29], oral cancer cells [30], hepatoma cells [31], human Ewing's tumor cells [32], prostate cancer cells [27], as well as glioblastoma cells [33]. Our data demonstrated a novel supplementation that quercetin inhibits both Hsp27 and COX-2 and eliminates the resistance to t-AUCB in glioblastoma.

\section{Conclusions}

Our study demonstrates that t-AUCB inhibits cell proliferation, migration and invasion and induces cell cycle G1 phase arrest in vitro; increased Hsp27 activation and following COX-2 overexpression confer resistance to $\mathrm{t}$-AUCB treatment in glioblastoma in vitro and in vivo; quercetin sensitizes glioblastoma to t-AUCB by dual inhibition of Hsp27 and COX-2. Combination of t-AUCB and quercetin may be a potential approach to treating glioblastoma.

\section{Abbreviations}

EETs: Epoxyeicosatrienoic acids; ARA: arachidonic acid; sEHIs: soluble epoxide hydrolase inhibitors; DHETs: dihydroxyeicosatrienoic acids; Hsp27: Heat shock protein 27; COX2: cyclooxygenase 2; DMSO: dimethyl sulfoxide; DMEM: Dulbecco's Modified Eagle's Medium; FBS: fetal bovine serum; CCK-8: cell counting kit-8; FITC: fluorescein isothiocyanate; PI: propidium iodide.

\section{Competing of interests}

The authors declare that they have no competing of interests.

\section{Authors' contributions}

$J \mathrm{~L}, \mathrm{CT}$ and $\mathrm{YF}$ designed the research; $\mathrm{L}, \mathrm{CT}, \mathrm{LL}$ and $\mathrm{RL}$ performed the research; $J L$ and CT analyzed the data; $J$ wrote the paper, YF revised the paper. All authors read and approved the final manuscript.

\section{Acknowledgements}

We thank Professor Bruce D. Hammock for providing the sEH inhibitor t-AUCB.

\section{Funding}

This study was supported by research fund from National Natural Science Foundation of China (NO.81301905)

\section{Author details}

'Department of Neurosurgery, Jinling Hospital, School of Medicine, Nanjing University, 305 East Zhongshan Road, Nanjing City 210002 Jiangsu Province, China. ${ }^{2}$ Department of Neurosurgery, Second Affiliated Hospital of Soochow University, 1055 Sanxiang Road, Suzhou 215004, China.

Received: 1 February 2016 Accepted: 21 March 2016

Published online: 02 April 2016

References

1. Qin LS, Jia PF, Zhang ZQ, Zhang SM. ROS-p53-cyclophilin-D signaling mediates salinomycin-induced glioma cell necrosis. J Exp Clin Cancer Res. 2015;34:57. doi:10.1186/s13046-015-0174-1.

2. Cheng Z, Wang HZ, Li X, Wu Z, Han Y, Li Y, et al. MicroRNA-184 inhibits cell proliferation and invasion, and specifically targets TNFAIP2 in Glioma. J Exp Clin Cancer Res. 2015;34:27. doi:10.1186/s13046-015-0142-9.

3. Zhu M, Chen L, Zhao P, Zhou H, Zhang C, Yu S, et al. Store-operated $\mathrm{Ca}(2+)$ entry regulates glioma cell migration and invasion via modulation of Pyk2 phosphorylation. J Exp Clin Cancer Res. 2014;33:98. doi:10.1186/s13046-014-0098-1.

4. Yan Y, Xu Z, Dai S, Qian L, Sun L, Gong Z. Targeting autophagy to sensitive glioma to temozolomide treatment. J Exp Clin Cancer Res. 2016;35:23. doi: 10.1186/s13046-016-0303-5.

5. Gao KM, Chen XC, Zhang JX, Wang Y, Yan W, You YP. A pseudogenesignature in glioma predicts survival. J Exp Clin Cancer Res. 2015;34:23. doi:10.1186/s13046-015-0137-6.

6. Li X, Liu C, Ip BC, Hu KQ, Smith DE, Greenberg AS, et al. Tumor progression locus 2 ablation suppressed hepatocellular carcinoma development by inhibiting hepatic inflammation and steatosis in mice. J Exp Clin Cancer Res. 2015:34:138. doi:10.1186/s13046-015-0254-2

7. Giordano G, Febbraro A, Tomaselli E, Sarnicola ML, Parcesepe P, Parente D, et al. Cancer-related CD15/FUT4 overexpression decreases benefit to agents targeting EGFR or VEGF acting as a novel RAF-MEK-ERK kinase downstream regulator in metastatic colorectal cancer. J Exp Clin Cancer Res. 2015;34:108. doi:10.1186/s13046-015-0225-7.

8. Li L, Xu L, Yan J, Zhen ZJ, Ji Y, Liu CQ, et al. CXCR2-CXCL1 axis is correlated with neutrophil infiltration and predicts a poor prognosis in hepatocellular carcinoma. J Exp Clin Cancer Res. 2015;34:129. doi:10.1186/s13046-015-0247-1.

9. Vera M, Barcia E, Negro S, Marcianes P, Garcia-Garcia L, Slowing K, et al. New celecoxib multiparticulate systems to improve glioblastoma treatment. Int J Pharm. 2014;473(1-2):518-27. doi:10.1016/j.jpharm.2014.07.028.

10. Zeldin DC. Epoxygenase pathways of arachidonic acid metabolism. J Biol Chemi. 2001:276(39):36059-62. doi:10.1074/jbc.R100030200.

11. Zhang G, Kodani S, Hammock BD. Stabilized epoxygenated fatty acids regulate inflammation, pain, angiogenesis and cancer. Prog Lipid Res. 2014; 53:108-23. doi:10.1016/j.plipres.2013.11.003.

12. Zhang G, Panigrahy D, Mahakian LM, Yang J, Liu JY, Stephen Lee KS, et al. Epoxy metabolites of docosahexaenoic acid (DHA) inhibit angiogenesis, tumor growth, and metastasis. Proc Natl Acad Sci U S A. 2013:110(16):6530-5. doi:10.1073/pnas.1304321110.

13. Wang D, Dubois RN. Eicosanoids and cancer. Nat Rev Cancer. 2010;10(3): 181-93. doi:10.1038/nrc2809.

14. Panigrahy D, Edin ML, Lee CR, Huang S, Bielenberg DR, Butterfield CE, et al. Epoxyeicosanoids stimulate multiorgan metastasis and tumor dormancy escape in mice. J Clin Invest. 2012;122(1):178-91. doi:10.1172/JCI58128. 
15. Li J, Hu W, Lan Q. The apoptosis-resistance in t-AUCB-treated glioblastoma cells depends on activation of Hsp27. J Neuro-Oncol. 2012;110(2):187-94. doi:10.1007/s11060-012-0963-8.

16. Zhang G, Panigrahy D, Hwang SH, Yang J, Mahakian LM, Wettersten HI, et al. Dual inhibition of cyclooxygenase-2 and soluble epoxide hydrolase synergistically suppresses primary tumor growth and metastasis. Proc Natl Acad Sci U S A. 2014;111(30):11127-32. doi:10.1073/pnas.1410432111.

17. Liu JY, Tsai HJ, Hwang SH, Jones PD, Morisseau C, Hammock BD. Pharmacokinetic optimization of four soluble epoxide hydrolase inhibitors for use in a murine model of inflammation. Br J Pharmacol. 2009;156(2): 284-96. doi:10.1111/j.1476-5381.2008.00009.x.

18. Xu K, Wang L, Shu HK. COX-2 overexpression increases malignant potential of human glioma cells through Id1. Oncotarget. 2014;5(5):1241-52.

19. Li JY, Li RJ, Wang HD. gamma-secretase inhibitor DAPT sensitizes t-AUCBinduced apoptosis of human glioblastoma cells in vitro via blocking the p38 MAPK/MAPKAPK2/Hsp27 pathway. Acta Pharmacol Sin. 2014;35(6):825-31. doi:10.1038/aps.2013.195.

20. Alam R, Schultz CR, Golembieski WA, Poisson LM, Rempel SA. PTEN suppresses SPARC-induced pMAPKAPK2 and inhibits SPARC-induced Ser78 HSP27 phosphorylation in glioma. Neuro-Oncology. 2013;15(4):451-61. doi:10.1093/neuonc/nos326.

21. Cheranov SY, Karpurapu M, Wang D, Zhang B, Venema RC, Rao GN. An essential role for SRC-activated STAT-3 in 14,15-EET-induced VEGF expression and angiogenesis. Blood. 2008;111(12):5581-91. doi:10.1182/blood-2007-11-126680.

22. Webler AC, Michaelis UR, Popp R, Barbosa-Sicard E, Murugan A, Falck JR, et al. Epoxyeicosatrienoic acids are part of the VEGF-activated signaling cascade leading to angiogenesis. Am J Physiol Cell Physiol. 2008;295(5): C1292-301. doi:10.1152/ajpcell.00230.2008.

23. Wu G, Luo J, Rana JS, Laham R, Sellke FW, Li J. Involvement of COX-2 in VEGF-induced angiogenesis via P38 and JNK pathways in vascular endothelial cells. Cardiovasc Res. 2006;69(2):512-9. doi:10.1016/j.cardiores. 2005.09.019

24. Lasa M, Mahtani KR, Finch A, Brewer G, Saklatvala J, Clark AR. Regulation of cyclooxygenase 2 mRNA stability by the mitogen-activated protein kinase p38 signaling cascade. Mol Cell Biol. 2000;20(12):4265-74.

25. Shoskes DA, Zeitlin SI, Shahed A, Rajfer J. Quercetin in men with category III chronic prostatitis: a preliminary prospective, double-blind, placebo-controlled trial. Urology. 1999;54(6):960-3.

26. Wang G, Wang JJ, Chen XL, Du SM, Li DS, Pei ZJ, et al. The JAK2/STAT3 and mitochondrial pathways are essential for quercetin nanoliposome-induced C6 glioma cell death. Cell Death Dis. 2013;4:e746. doi:10.1038/cddis.2013.242.

27. Yang F, Song L, Wang H, Wang J, Xu Z, Xing N. Combination of Quercetin and 2-Methoxyestradiol Enhances Inhibition of Human Prostate Cancer LNCaP and PC-3 Cells Xenograft Tumor Growth. PLoS One. 2015;10(5): e0128277. doi:10.1371/journal.pone.0128277.

28. Hsu HS, Lin JH, Huang WC, Hsu TW, Su K, Chiou SH, et al. Chemoresistance of lung cancer stemlike cells depends on activation of Hsp27. Cancer. 2011; 117(7):1516-28. doi:10.1002/cncr.25599.

29. Lee $\mathrm{CH}$, Hong HM, Chang YY, Chang WW. Inhibition of heat shock protein (Hsp) 27 potentiates the suppressive effect of Hsp90 inhibitors in targeting breast cancer stem-like cells. Biochimie. 2012;94(6):1382-9. doi:10.1016/j. biochi.2012.02.034.

30. Chen SF, Nieh S, Jao SW, Liu CL, Wu CH, Chang YC, et al. Quercetin suppresses drug-resistant spheres via the p38 MAPK-Hsp27 apoptotic pathway in oral cancer cells. PLoS One. 2012;7(11):e49275. doi:10.1371/journal.pone.0049275.

31. Sharma A, Upadhyay AK, Bhat MK. Inhibition of Hsp27 and Hsp40 potentiates 5 -fluorouracil and carboplatin mediated cell killing in hepatoma cells. Cancer Biol Ther. 2009;8(22):2106-13.

32. Debes A, Oerding M, Willers R, Gobel U, Wessalowski R. Sensitization of human Ewing's tumor cells to chemotherapy and heat treatment by the bioflavonoid quercetin. Anticancer Res. 2003;23(4):3359-66.

33. Sang DP, Li RJ, Lan Q. Quercetin sensitizes human glioblastoma cells to temozolomide in vitro via inhibition of Hsp27. Acta Pharmacol Sin. 2014 35(6):832-8. doi:10.1038/aps.2014.22.

\section{Submit your next manuscript to BioMed Central and we will help you at every step:}

- We accept pre-submission inquiries

- Our selector tool helps you to find the most relevant journal

- We provide round the clock customer support

- Convenient online submission

- Thorough peer review

- Inclusion in PubMed and all major indexing services

- Maximum visibility for your research

Submit your manuscript at www.biomedcentral.com/submit
Biomed Central 